\title{
Effect of Intermittent Blood Volume Fluctuation of Light Resistance Exercise after Ingestion of the High-Protein Snacks on Plasma Branched-Chain Amino Acid Concentrations in Young Adults
}

\author{
Yushi KATO ${ }^{1}$, Shigeharu NumaO ${ }^{2}$, Ryoko MiYauchi ${ }^{1}$ and Masashige SuzUKI ${ }^{2, *}$ \\ ${ }^{1}$ Graduate School of Sport Sciences, and ${ }^{2}$ Faculty of Sport Sciences, Waseda University, Tokorozawa, \\ Saitama 359-1192, Japan
}

(Received February 10, 2010)

\begin{abstract}
Summary The study investigated exercise patterns resulting in the more efficient promotion of amino acid utilization. High-protein snacks (HPS; $15 \mathrm{~g}$ protein, $18 \mathrm{~g}$ sugar) were ingested by 8 young adult subjects $3 \mathrm{~h}$ after the basal meal ingestion. Sixty minutes after the HPS ingestion, the subjects performed arm flex/extend exercises for $15 \mathrm{~min}$. The difference between 2 exercise patterns was compared. Pattern 1: High-number long-interval (HL) arm flex/extend $(3+3 \mathrm{~s})$ exercise; the HL group performed 9 sets of 15 exercises with a $10 \mathrm{~s}$ interval between sets. Pattern 2: Low-number short-interval (LS) arm flex/extend ( $3+3 \mathrm{~s})$ exercise; the LS group performed 27 sets of 5 exercises with a 3-4 s interval between sets (135 exercises during $15 \mathrm{~min}$, respectively). The plasma branched-chain amino acid (BCAA) concentrations were measured before the HPS ingestion, before the exercise, and 60 and 90 min after the HPS ingestion. The plasma BCAA concentrations increased significantly after the HPS ingestion. In the HL group, BCAA concentration increased consistently during the period and 60 to 90 min after the HPS ingestion. During the same period in the LS group the BCAA concentration stopped increasing. After HPS ingestion, a significantly greater suppressive effect on plasma BCAA concentration was seen in the LS group compared to the HL group. Results confirmed that the intermittent blood volume fluctuation in muscle tissue during the exercise pattern performed by the LS group had an effect on the utilization of nutritional components (BCAA, glucose) from the blood, and showed the possibility that the group where the blood volume in the muscle tissue increased/lowered with higher frequency was a more effective exercise pattern for nutrient utilization.
\end{abstract}

Key Words high-protein snack, light resistance exercise, branched-chain amino acids, muscular blood volume

The ingestion of the high-protein snacks (HPS) around $3 \mathrm{~h}$ after the basal meals were found to result in the efficient delivery of amino acids to peripheral tissues such as muscle in glucocorticoid-injected rats $(1,2)$. The performance of voluntary tower climbing exercise (3) as light-resistance exercise after ingestion of the HPS, producing intermittent blood volume fluctuation in muscle tissue, resulted in increased muscle mass in glucocorticoid-injected rats. In addition, light-resistance exercise (dumbbell exercise) during a period of elevated plasma branched-amino acid (BCAA) concentration following the HPS which was taken $3 \mathrm{~h}$ after the basal meals seemed also effective in promoting the utilization of BCAA in human subjects (4).

Performing dumbbell exercise in half-crouching position, with the upper body angled forwards, grasping the dumbbells firmly, twisting the wrists inward, and keeping them curled $(5,6)$ resulted in reduced blood volume in the antebrachial and femoral muscles during exercise and sudden increase in blood volume during the interval period between exercise sets (4). It is thought

\footnotetext{
*To whom correspondence should be addressed.
}

E-mail: suzuki-dumbbell@waseda.jp that the climbing exercise in rats also causes intermittent blood volume fluctuation in muscle tissues, similar to that which arises during dumbbell exercise in human subjects (4).

The delivery of a large volume of amino acids to muscles on the ingestion of the HPS is influenced by the intermittent blood volume fluctuation in muscle tissue that occurs during light resistance exercise. As the efficient uptake of amino acids by muscle tissue is thought to contribute to greater increase in both muscle mass and muscle strength, the intermittent blood volume fluctuation in muscle tissue can be considered to control how efficiently amino acids and other nutritional components in the blood are taken up by the muscle.

This study investigates different patterns (frequency of exercises per set, and length and frequency of intervals) of varying blood volume in muscular tissue, the effect of these differences on BCAA and glucose concentrations in blood plasma, and investigates the pattern of blood volume fluctuation resulting in the more effective utility of amino acids and glucose.

\section{SUBJECTS AND METHODS}

Subjects. Eight healthy young adults ( 3 males and 5 
Table 1. The characteristics of the subjects.

\begin{tabular}{lr}
\hline Age $(\mathrm{y})$ & $24.5 \pm 4.6$ \\
Height $(\mathrm{cm})$ & $168.1 \pm 7.6$ \\
Weight $(\mathrm{kg})$ & $58.7 \pm 7.6$ \\
BMI $\left(\mathrm{kg} / \mathrm{m}^{2}\right)$ & $20.7 \pm 1.2$ \\
\hline
\end{tabular}

Values are means \pm SD $(n=8)$.

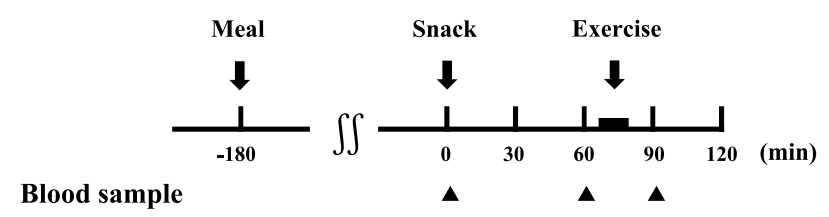

Fig. 1. Experimental protocol.

females) participated in this study (Table 1). They were randomly assigned to either of 2 groups as follows: High-number long-interval (HL group) and Low-number short-interval (LS group).

Seven days after the first experiment, they were crossed over to the opposite intervention. The subjects gave their consent regarding the purpose of the study, study methodology, and publication of the study results. This study was implemented with permission from the Waseda University Ethics Committee.

Experimental protocol. The subjects were prohibited from eating or drinking excessively and doing exercise on the day before the experiment. Eating or drinking, except for water, was prohibited for $12 \mathrm{~h}$ prior to the start of the experiment.

On the experimental day, the subjects were provided with the breakfast (basal meal) and the HPS $3 \mathrm{~h}$ after the basal meal. The subjects performed arm flex/extend exercises for $15 \mathrm{~min}$, $60 \mathrm{~min}$ after the HPS ingestion. Blood was collected before the HPS ingestion (at 0 min) and at 60 and 90 min after the HPS ingestion (Fig. 1).

Breakfast (Basal meal). Composition of basal meal is toast, milk, cornflakes, cheese, ham, orange juice, and fruit jelly. The energy and protein content of breakfast were calculated by equally dividing daily total energy (male, $42 \mathrm{kcal} \cdot \mathrm{kg}^{-1} \cdot \mathrm{d}^{-1}$; female, $35 \mathrm{kcal} \cdot \mathrm{kg}^{-1} \cdot \mathrm{d}^{-1}$ ) and protein (male and female, $1.0 \mathrm{~g} \cdot \mathrm{kg}^{-1} \cdot \mathrm{d}^{-1}$ ) into three, after subtracting the portion of the HPS.

High-protein snacks. The HPS were made of dried egg whites (51 kcal/14.5 g) (Kewpie Corporation, Tokyo, Japan), gelatin $(2.5 \mathrm{~g})$, sugar $(18.0 \mathrm{~g})$ and water and consisted of $15 \mathrm{~g}$ protein and an energy content of 130 kcal.

The amounts of the protein of the HPS were determined in our previous study (4).

Exercise. Human subjects grasped the dumbbells firmly, twisted the wrists inward and kept them curled, and with wrists facing in towards the body, flexed arms towards the shoulders and extended them out again. This arms flex/extend exercise was performed for $15 \mathrm{~min}$. Two exercise patterns were set as follows: Pattern 1: High-number long-interval (HL) arm flex/ extend $(3+3 \mathrm{~s})$ exercise; the HL group performed 9 sets
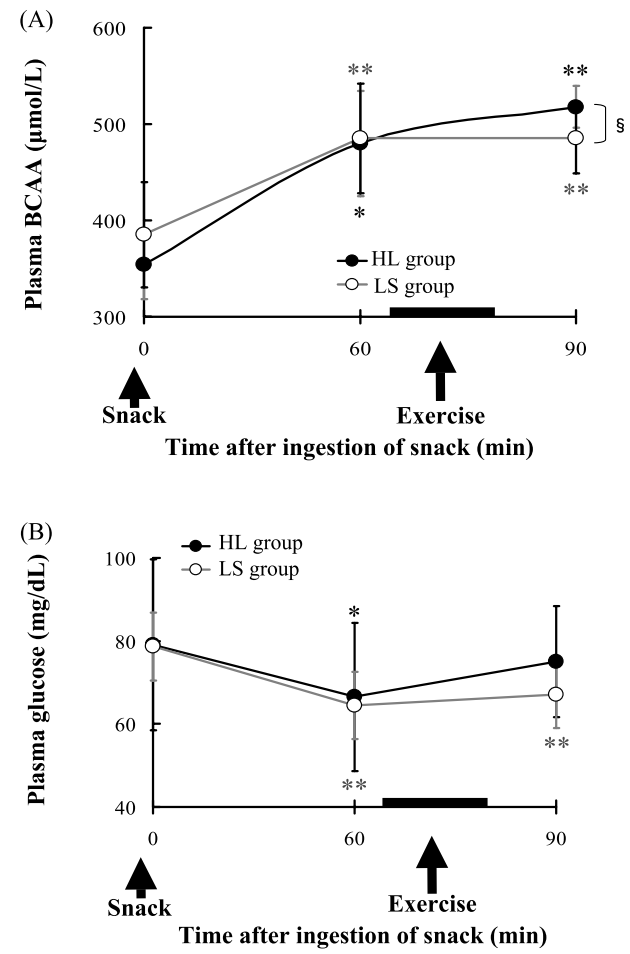

Fig. 2. Responses of plasma BCAA (A) and glucose (B), concentrations to arm flex/extend exercise after the ingestion of the HPS. Values are means \pm SD $(n=8)$. ${ }^{*} p<0.05,{ }^{* *} p<0.01$ : Significantly different from time $0 .{ }^{\S}<0.05$ : Significantly different among 2 groups.

of 15 exercises with a $10 \mathrm{~s}$ interval between sets. Pattern 2: Low-number short-interval (LS) arm flex/extend $(3+3 \mathrm{~s})$ exercise; the LS group performed 27 sets of 5 exercises with a 3-4 s interval between sets (135 exercises during $15 \mathrm{~min}$, respectively).

Subjects received prior training on the correct pose to assume during flexing and extension of the arms, correct movement, and at what speed to conduct the exercise. Subjects approached the experiments with sufficient mastery of the exercise.

Analysis of blood components. Plasma was analyzed for BCAA and glucose by enzymatic methods (BCAA: leucine dehydrogenase and diaphorase, glucose: glucokinase and glucose 6-phophatase) in Mitsubishi Chemical Medience, Co. Ltd. (Tokyo, Japan).

Muscle blood volume. Changes in total hemoglobin content in the antebrachial muscle was measured during the dumbbell exercises using near infrared spectroscopy (Hamamatsu Photonics, Co. Ltd., Shizuoka, Japan) to confirm the fluctuation in skeletal muscle blood volume (7).

Statistical analysis. All data were expressed as means \pm SD. Analysis of variance was performed on time ( 3 time points measured) and experimental conditions (2 exercise patterns), and Huynh-Feldt correction was applied to the degrees of freedom to reduce the risk of Type I errors when Mauchly's sphericity could not be assumed. The least significant difference (LSD) was used to compare multiple values.

A paired $t$-test was also performed to compare 

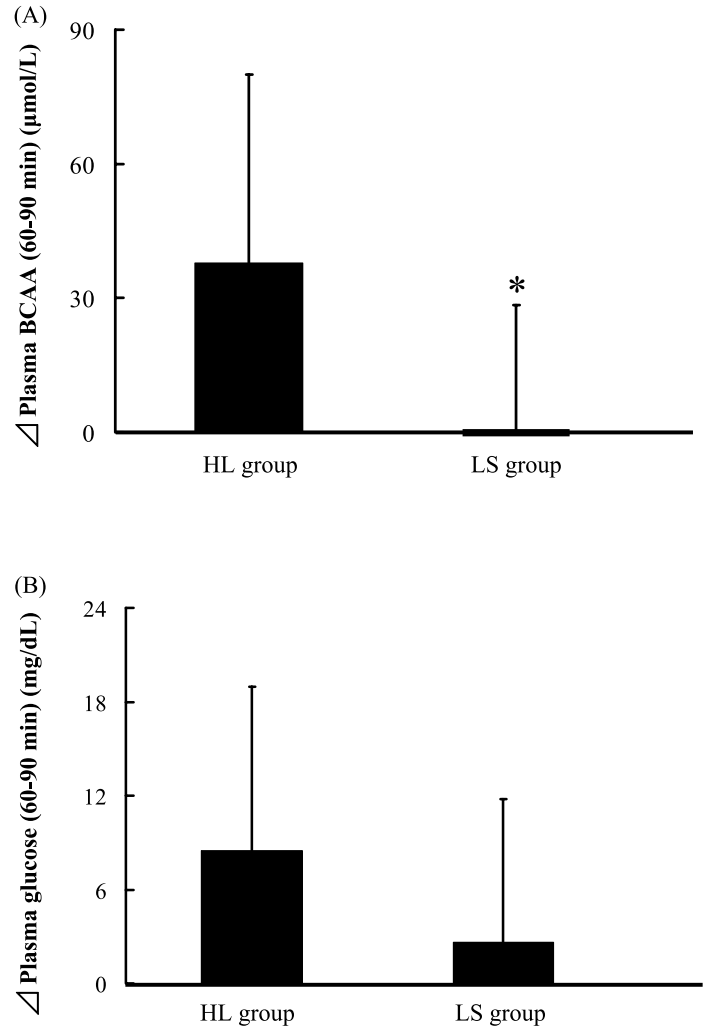

Fig. 3. Changes in plasma BCAA (A) and glucose (B), concentrations after arm flex/extend exercise at 60 90 min after ingestion of the HPS. Values are means \pm SD $(n=8) .{ }^{*} p<0.05$ : Significantly different from the HL group.

changes in concentrations of plasma components between 60 and 90 min after the HPS ingestion. Statistical processing was performed by statistical analysis software (SPSS15.0J, SPSS Japan), using a 5\% level of significance in all cases.

\section{RESULTS}

\section{Plasma BCAA concentrations}

Plasma BCAA concentrations were significantly higher at 60, and 90 min after the HPS ingestion, compared with the point immediately before the HPS ingestion (at $0 \mathrm{~min}$ ), in both the HL and the LS groups. In the HL group, plasma BCAA concentration continually increased for $90 \mathrm{~min}$ after the HPS ingestion. In the LS group, plasma BCAA concentrations stopped increasing during the arms flex/extend exercise (Fig. 2A). There was a significant difference in plasma BCAA concentrations in the LS group compared with the HL group between 60 and $90 \mathrm{~min}$ (Fig. 3A).

Plasma glucose concentrations

Plasma glucose concentrations decreased $60 \mathrm{~min}$ after the HPS ingestion. The subsequent rapid decrease of the concentrations until $60 \mathrm{~min}$ was followed by the increase again in both groups (Fig. 2B). Changes in plasma glucose concentrations after arms flex/extend exercise 60-90 min after the HPS ingestion in the LS group compared with the HL group was not significantly different (Fig. 3B).
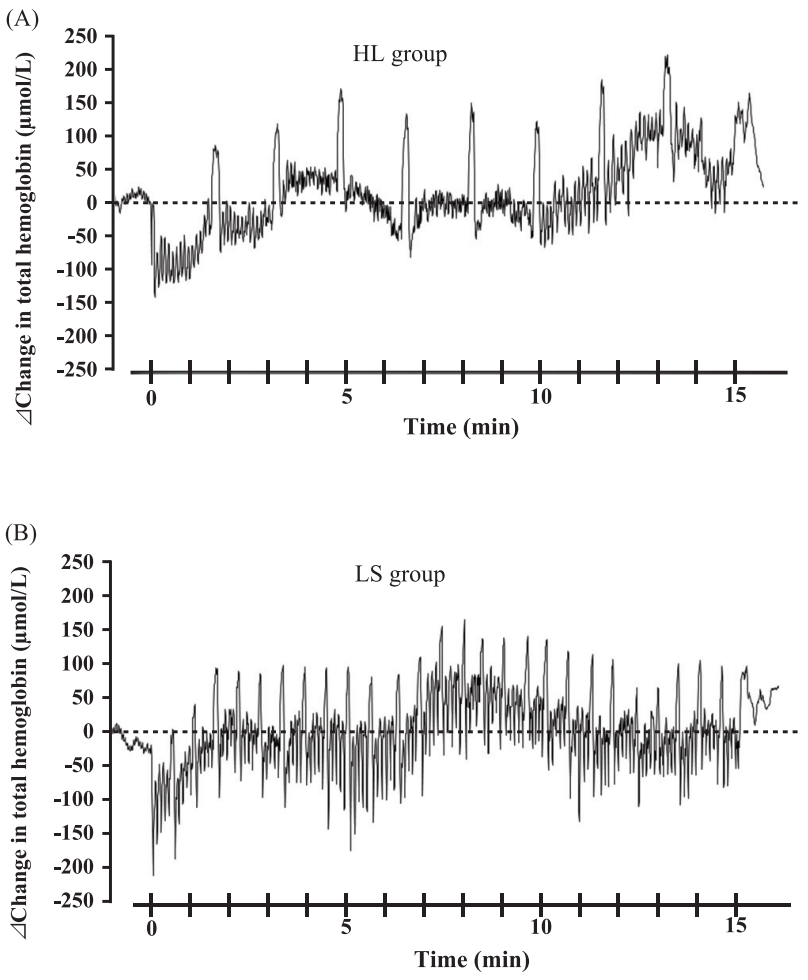

Fig. 4. Changes in total hemoglobin contents in the antebrachial muscle during arm flex/extend exercise. (A) HL group. (B) LS group. Values are means \pm SD $(n=8)$.

\section{Muscle blood flow}

For both the HL and the LS exercise patterns, total hemoglobin in the antebrachial muscle tissue fell markedly during flex/extend of the arm, and increased suddenly at the interval. The number of times total hemoglobin suddenly increased and decreased was equivalent to the number of repeated exercise sets, with hemoglobin increase and decrease occurring more often in the LS group with more exercise sets performed (Fig. 4).

The wave height (periodic fluctuation) in total hemoglobin during the transfer from interval to next exercise set was similar between the HL and the LS groups. The wave heights in total hemoglobin during exercises were alike among the 2 groups.

\section{DISCUSSION}

As amino acids derived from proteins that are consumed in the basal meals are mainly used in the small intestine and liver $(8)$, we focus here on the difficulty peripheral tissue such as muscles have in using amino acids. In a study of a muscle loss model in glucocorticoid-injected rats, the HPS were ingested $3 \mathrm{~h}$ after the basal meals, amino acid concentrations rose in the peripheral blood, and simultaneous to this increase, light resistance exercise was performed $(1,2)$. The study showed intermittent blood volume fluctuation in muscular tissue can be effective in muscle uptake of amino acids. Kato et al. reported the ingestion of the HPS $3 \mathrm{~h}$ after the basal meals and performing light resistance exercise (dumbbell exercise) under conditions of ele- 
vated amino acid concentration in the blood may be effective in promoting utilization of amino acids in humans (4). From these results, intermittent blood volume fluctuation in muscular tissue through exercise can be regarded as controlling the efficiency of muscle uptake of nutritional components in the blood (i.e., glucose, amino acids). In this study we examined different patterns of elevated then reduced blood volume in muscle tissue during arm flex/extend exercises after the ingestion of the HPS and the effects of these patterns on plasma BCAA concentrations, and searched for an exercise pattern resulting in a greater promotional efficiency for BCAA utilization in muscle tissue.

Two changing patterns of blood volume in the muscle were investigated by comparing 2 subject groups each performing different arm flex/extend exercise routines. The speed of the arm flex and arm extend movement was performed slowly and over a period of $3 \mathrm{~s}$ and $3 \mathrm{~s}$. Subjects in the HL group performed 9 sets of 15 arm flex/extend exercises with a 10-s interval between sets. Subjects in the LS group performed 27 sets of 5 arm flex/extend exercises with a 3-4 s interval between sets. The speed of arm flex/extend exercises and the number of arm flex/extend exercises were identical in both groups $(3+3 \mathrm{~s}$, and 135 exercises, respectively). The exercises were set to be completed in $15 \mathrm{~min}$ in both the HL group and the LS group.

Plasma BCAA concentration increased significantly until 60 min after ingestion of the HPS in both groups. Between $60 \mathrm{~min}$ and $90 \mathrm{~min}$ after the HPS ingestion (after exercise completion), plasma BCAA concentration continued to increase in the HS group, while in the LS group the increase stopped, and a significant difference was shown between the 2 groups.

Many reports exist of studies into the effects of the timing of exercising and protein or amino acid administration on the metabolism of muscle protein (9-14). In experiments where protein was administered to human subjects immediately after and $2 \mathrm{~h}$ after resistance exercise, administering protein immediately after resistance exercise had the greater effect in terms of muscle enlargement and muscle strengthening (9). In experiments where a mixture of essential amino acids and sugar was administered to human subjects, compared to administering them immediately after exercise, administration immediately before exercise gave a markedly greater total amino acid uptake in the muscles of the lower limbs, as well as greater net protein synthesis (14). Matsumoto et al. have reported the plasma BCAA uptake after BCAA supplement ingestion during exercise of $50 \%$ maximum intensity on a cycling ergometer with elevated concentrations of plasma BCAA (15). One study also reports the endogenous suppression of protein degradation on oral administration of BCAA before exercise, resulting in suppression of protein degradation during exercise, increased ammonia production in muscles, and reduced quantities of essential amino acids released from muscles (16). From these results we may regard the ingestion of essential amino acids and sugar before exercise as maximizing amino acid uptake by increasing the blood volume in the muscles during exercise, and effectively promoting muscle protein synthesis.

Bohé et al. repeated the stimulatory effect of essential amino acids on muscle protein in human subjects, reporting the stimulatory effect was limited to a 90-min period following elevation of plasma amino acid concentration (17). Bohé et al. also reported the stimulation of muscle protein synthesis in humans is regulated by the concentration of essential amino acids in the blood, and elevation of this concentration by 40-80\% has the greatest effect in stimulating muscle protein synthesis (18). In other words, maintaining the concentration of essential amino acids at a level elevated by $40-80 \%$ continuously for a period of 90 min can be regarded as producing the most stimulatory conditions for muscle protein synthesis possible. In this study, the HPS ingestion maintained the concentration of BCAA elevated by close to $40 \%$ until $90 \mathrm{~min}$ after the HPS ingestion, and showed that the HPS ingestion may stimulate muscle protein synthesis in humans.

The increase and decrease of blood in active muscles occurs as a result of the interaction of a number of different factors. It is reported that because exercise involving bending of the joints requires the contraction of muscle fibers, volume of blood inflow to muscles is restricted to no more than that which occurs during static exercise maintaining a constant tensile force (19). In this study we measured the cyclic variation of total hemoglobin in antebrachial muscle tissue during exercise and found that starting arm flex/extend exercises while the muscle was tense caused a sudden reduction in hemoglobin levels, confirming that the increase/ decrease in hemoglobin during exercise is restricted. This study also found total hemoglobin levels increased sharply during the period of muscle relaxation at intervals between exercises, indicating increased blood volume in the muscle tissue.

During the 15 min of exercises performed by human subjects in this study, 3 times as many muscle relaxation intervals were present in the LS pattern compared to the HL pattern (LS: 27, HL: 9). As the volume of blood in the muscle reaches its highest value around 3$4 \mathrm{~s}$ into a period of muscular relaxation, the greatest volume for blood in the muscle can be regarded as having been reached more frequently in the LS group. This effect is believed to have favored the LS group over the HL group in terms of promoting muscle uptake of BCAA, suppressing the increase in blood plasma concentration of BCAA, and resulting in significantly different cyclical variations in plasma BCAA concentrations between the 2 groups.

Though the change in glucose concentration before and after exercise was not significant in either group, the increase in blood glucose concentration was suppressed in the LS group when compared to the HL group. The uptake of glucose from the blood and its metabolic utilization by muscle tissue can be regarded as being promoted in a similar way to that which occurs for BCAA in the blood. 
The above results confirm that the pattern of blood volume variation presented in the LS group, compared to that of the HL group, resulted in the effective promotion of utilization of nutritional components in the blood (BCAA, glucose). Changing the interval pattern and number of exercise repeats per set while keeping other factors constant (15 min of exercises, same number of exercises, same speeds), and so changing the pattern of variation of blood in the muscle tissue, was confirmed to effect muscle utilization of nutritional components in the blood.

The current study has not measured the muscle tissue uptake of amino acids, and the definite mechanism of the process remains unknown. Future research will therefore verify the patterns of variation of blood volume in muscular tissue that promote the efficient utilization of nutritional components. Elucidation of the mechanisms involved in this process should lead to applications in the prevention of sarcopenia, as well as in sports.

\section{REFERENCES}

1) Matsuo T, Suzuki M. 2004. Effects of voluntary resistance exercise and high-protein snack on hind-limb muscle mass and protein synthesis in rats given glucocorticoid-injections. Health Sci 20: 275-283.

2) Matsuo T, Suzuki M. 2005. Effects of resistance exercise and high-protein snacks consisting of different proteins on muscle mass and protein synthesis in rats given glucocorticoid-injections. Health Sci 21: 246-256.

3) Notomi T, Okimoto N, Okazaki Y, Tanaka Y, Nakamura Y, Suzuki M. 2001. Effects of tower climbing exercise on bone mass, strength, and turnover in growing rats. $J$ Bone Miner Res 16: 166-174.

4) Kato Y, Sawada A, Numao S, Miyauchi R, Imaizumi K, Sakamoto S, Suzuki M. 2009. Effect of light resistance exercise after ingestion of a high-protein snack on plasma branched-chain amino acid concentrations in young adult females. J Nutr Sci Vitaminol 55: 106-111.

5) Suzuki M. 2003. Health promotion by dumbbell exercise. Bull Int Health Sport Sci Univ Tsukuba 26: 1-6 (in Japanese).

6) Matsuo T, Suzuki M. 1999. Effect of dumb-bell exercise with and without energy restriction on resting metabolic rate, diet-induced thermogenesis and body composition in mildly obese women. Asia Pac J Clin Nutr 8: 136-141.

7) De Blasi RA, Ferrari M, Natali A, Conti G, Mega A, Gasparetto A. 1994. Noninvasive measurement of forearm blood flow and oxygen consumption by near-infrared spectroscopy. J Appl Physiol 76: 1388-1393.
8) Boirie Y, Gachon P, Beaufrere B. 1997. Splanchnic and whole-body leucine kinetics in young and elderly men. Am J Clin Nutr 65: 489-495.

9) Esmarck B, Andersen JL, Olsen S, Richter EA, Mizuno M, Kjaer M. 2001. Timing of postexercise protein intake is important for muscle hypertrophy with resistanse training in elderly humans. J Physiol 535: 301-311.

10) Tipton KD, Rasmussen BB, Miller SL, Wolf SE, OwensStovall SK, Petrini BE, Wolfe RR. 2001. Timing of amino acid-carbohydrate ingestion alters anabolic response of muscle to resistance exercise. Am J Physiol Endocrinol Metab 281: E197-E206.

11) Okamura K, Doi T, Hamada K, Sakurai M, Matsumoto K, Imaizumi K, Yoshioka Y, Shimizu S, Suzuki M. 1997. Effect of amino acid and glucose administration during postexercise recovery on protein kinetics in dogs. Am J Physiol Endocrinol Metab 272: E1023-E1030.

12) Suzuki M, Doi T, Lee SJ, Okamura K, Shimizu S, Okano G, Sato Y, Shimomura Y, Fushiki T. 1999. Effect of meal timing after resistance exercise on hindlimb muscle mass and fat accumulation in trained rats. J Nutr Sci Vitaminol 45: 401-409.

13) Cribb PJ, Hayes A. 2006. Effects of supplement timing and resistance exercise on skeletal muscle hypertrophy. Med Sci Sports Exerc 38: 1918-1925.

14) Tipton KD, Rasmussen BB, Miller SL, Wolf SE, OwensStovall SK, Petrini BE, Wolfe RR. 2001. Timing of amino acid-carbohydrate ingestion alters anabolic response of muscle to resistance exercise. Am J Physiol Endocrinol Metab 281: E197-E206.

15) Matsumoto K, Mizuno M, Mizuno T, Dilling-Hansen B, Lahoz A, Bertelsen V, Munster H, Jordening H, Hamada K, Doi T. 2007. Branched-chain amino acids and arginine supplementation attenuates skeletal muscle proteolysis induced by moderate exercise in young individuals. Int J Sports Med 28: 531-538.

16) MacLean DA, Graham TE, Saltin B. 1994. Branchedchain amino acids augment ammonia metabolism while attenuating protein breakdown during exercise. Am J Physiol Endrocrinol Metab 267: E1010-E1022.

17) Bohé J, Low JFA, Wolfe RR, Rennie MJ. 2001. Latency and duration of stimulation of human muscle protein synthesis during continuous infusion of amino acids. $J$ Physiol 532: 575-579.

18) Bohé J, Low A, Wolfe RR, Rennie MJ. 2003. Human muscle protein synthesis is modulated by extracellular, not intramuscular amino acid availability: a doseresponse study. J Physiol 552: 315-324.

19) Robergs RA, Icenogle MV, Hudson TL, Greene ER. 1997. Temporal inhomogeneity in brachial artery blood flow during forearm exercise. Med Sci Sports Exerc 29: 10211027. 